\title{
Avaliação das Concentraçōes Séricas dos Peptídeos Natriuréticos Vasoativos ANP e BNP, nos Fetos de Mães Hipertensas e Normotensas
}

Autor: Mário Dias Corrêa Junior

Orientador: Prof. Dr. Mário Jorge Barreto Viegas de Castro

Dissertação de Mestrado apresentada ao Programa de Pós-Graduação em Ginecologia e Obstetrícia da Faculdade de Medicina da UFMG, em 30 de agosto de 2002.

Objetivo: avaliar as concentrações séricas dos peptídeos natriuréticos, ANP e BNP, nos fetos de gestantes normotensas e hipertensas. Verificar se as concentrações séricas fetais do ANP e BNP são influenciadas pela hipertensão arterial materna ou pelo crescimento intra-uterino restrito (CIUR).

Pacientes e Métodos: foram incluídas no estudo 24 gestantes normotensas, 60 gestantes que desenvolveram pré-eclâmpsia pura ou associada à hipertensão arterial crônica. Todas as pacientes foram submetidas à cesariana e logo após o parto foi colhida uma amostra de sangue das artérias umbilicais, para a dosagem do peptídeo atrial natriurético (ANP) e do peptídeo natriurético do tipo B (BNP).

Resultados: os recém-nascidos do grupo das hipertensas apresentaram o BNP significativamente mais alto do que os recém-nascidos do grupo das normotensas $(p=0,025)$. Com relação ao ANP não houve diferença significativa entre os grupos $(p=0,19)$. Houve uma correlação positiva entre as concentrações séricas do ANP $(p=0,03)$ e BNP $(p=0,02)$ fetais e a pressão arterial média (PAM) das mães. Essa correlação desapareceu quando os fetos com CIUR e suas mães foram retirados da análise.

Conclusões: a hipertensão materna não influencia diretamente a produção do ANP e BNP fetais, mas pode influenciar indiretamente quando produz uma lesão placentária suficiente para levar ao desenvolvimento de CIUR.

Palavras-chave: Fator natriurético atrial. Préeclâmpsia. Restrição de crescimento fetal.

Autor: Ricardo de Carvalho Cavalli

Orientador: Prof. Dr. Sérgio Pereira da Cunha

Dissertação de Mestrado apresentada ao Departamento de Ginecologia e Obstetrícia da Faculdade de Medicina de Ribeirão Preto da Universidade de São Paulo, em 2 de agosto de 2002

A doença hipertensiva específica da gestação (DHEG) e a restrição de crescimento intra-útero (RCIU) associam-se diretamente às taxas de morbimortalidade materna e perinatal. A presença de exame dopplervelocimétrico indicando incisura das artérias uterinas entre a $24^{\text {a }}$ e a $26^{\text {a }}$ semana de gestação, vem se afirmando como screening positivo para DHEG e RCIU. A apoptose tem sido descrita em placentas de gestantes normais e está aumentada tanto em placentas de gestantes com DHEG quanto naquelas associadas à RCIU. Entretanto, não está estabelecido se o aumento da taxa de apoptose observada na DHEG e na RCIU é o resultado de um processo patológico instalado ou um componente etiológico no desenvolvimento destas complicações obstétricas. Estudos sobre os fatores envolvidos na regulação da apoptose referem que o $\mathrm{Bcl}-2$ é um dos inibidores deste processo. Os objetivos deste trabalho foram avaliar a influência da alterações do exame dopplervelocimétrico das artérias uterinas so- bre o padrão de apoptose e a expressão do $\mathrm{Bcl}-2 \mathrm{em}$ placentas de gestantes primigestas. A casuística deste estudo foi composta de 38 gestantes primigestas acompanhadas no HC-FMRPUSP. As mesmas foram avaliadas quanto aos parâmetros epidemiológicos e na $24^{\text {a }}$ semana de gestação foram submetidas ao exame dopplervelocimétrico das artérias uterinas e divididas em dois grupos. O Grupo DN foi constituído de 17 gestantes com exame dopplervelocimétrico normal e Grupo DA, constituído de 21 gestantes com exame dopplervelocimétrico alterado. Todas as pacientes tiveram seus partos realizados no HC-FMRPUSP. Após o parto a placentas foram coletadas e submetidas à avaliação macroscópica e microscópica segundo protocolos vigentes. As placentas foram submetidas à avaliação imunohistoquímica para verificação da taxa de apoptose (técnica do TUNEL) e da expressão do Anticorpo Monoclonal; Bcl-2. A avaliação dos parâmetros epidemiológicos maternos e neonatais demonstrou que os 
grupos eram homogêneos e comparáveis. A avaliação macroscópica das placentas, não evidenciou diferença estatística entre os grupos. Em relação a análise microscópica, verificou-se a tendência de uma maior taxa de alterações isquêmicas no grupo DA, porém sem diferença estatística significativa entre os grupos. O padrão apoptótico do grupo DN, foi de $0,17 \pm 0,10 \%$ e no grupo DA, $0,18 \pm 0,11 \%$ sem diferença estatística entre os grupos, não havendo influência relacionada à cor da paciente ou à via de parto. A expressão de Bcl-2 nas placentas do grupo DN e DA apresentou-se positivos em todos os casos. Constatou-se no grupo DN taxa de apoptose compativel com resultados de placentas nor- mais a termo descritos na literatura. O grupo DA com exame dopplervelocimétrico das artérias uterinas alterado não apresentou diferença estatística significativa nas taxas de apoptose em relação ao grupo DN. O padrão apoptótico aumentado na DHEG e no RCIU, provavelmente está associado ao padrão de hipóxia gerada pelo processo patológico instalado e não está associado ao padrão hipóxia envolvido na gênese das patologias acima mencionadas.

Palavras-chave: Apoptose. Pré-eclâmpsia. Restrição do crescimento intra-útero. Placenta.

\section{Expressão do P63 em Tumores Epiteliais do Ovário: uma Nova Evidência da Origem Alternativa dos Tumores Mucinosos}

Autor: Omero Benedicto Poli Neto

Orientador: Prof. Dr. Francisco José Candido dos Reis

Tese de Doutorado apresentada ao Departamento de Ginecologia e Obstetrícia da Faculdade de Medicina de Ribeirão Preto da Universidade de São Paulo, em 31 de outubro de 2003

\section{Resumo}

Objetivos: avaliar a expressão do P63 em tumores ovarianos epiteliais e verificar sua associação com os tipos histológicos.

Métodos: estudamos a expressão do P63 usando um anticorpo monoclonal (clone A4A) que reconhece todas as isoformas da proteína p63. Foram incluídos 91 espécimes de cistadenomas ovarianos: 29 cistadenomas mucinosos e 62 cistadenomas serosos; e 29 espécimes de carcinomas ovarianos: 3 tumores borderline mucinosos, 3 tumores borderline serosos, 1 carcinoma seroso, 17 carcinomas serosos e 5 carcinomas de outros subtipos histológicos.

Resultados: 85,7\% dos cistadenomas e 50\% dos tumores borderline apresentaram expressão do P63. Entretanto, uma minoria $(8,7 \%)$ de carcinomas p63-positivos foi identificada $(\mathrm{p}<0,0001)$. Todos os 3 tumores borderline p63-positivos eram tumores mucinosos. $\mathrm{O}$ espécime de carcinoma mucinoso foi p63-positivo e o outro carcinoma p63-positivo foi um carcinoma seroso pouco diferenciado estadio IV. Oito cistadenomas mucinosos $(27,6 \%)$ e somente 5 cistadenomas serosos $(8,1 \%)$ foram p63-negativos $(\mathrm{p}=0,02)$.

Conclusão: estes resultados mostram que cistadenomas ovarianos são mais freqüentemente p63positivos que tumores borderline e carcinomas ovarianos. Todavia, mais cisadenomas mucinosos p63-negativos foram identificados que cistadenomas serosos. Além do mais, tumores mucinosos borderline e invasores foram p63-positivos em contraste aos serosos borderline e invasores, sugerindo que a expressão do P63 pode sofrer influência tanto da natureza quanto do tipo histológico da lesão.

Palavras-chave: P63. Ovário: câncer. Ovário: cistos oncogenes. 